\title{
Analytical regularization based analysis of a spherical reflector symmetrically illuminated by an acoustic beam
}

\author{
Sergey S. Vinogradov ${ }^{\text {a) }}$ and Elena D. Vinogradova ${ }^{\text {b) }}$ \\ Department of Mathematics, University of Dundee, Dundee DD1 4HN, Scotland, United Kingdom
}

Alexander I. Nosich

Institute of Radiophysics and Electronics of the National Academy of Sciences, Ul. Proskury 12, Kharkov 310085, Ukraine

Ayhan Altintaş
Department of Electrical and Electronics Engineering, Bilkent University, 06533 Ankara, Turkey

(Received 3 April 1999; revised 16 February 2000; accepted 18 February 2000)

\begin{abstract}
A mathematically accurate and numerically efficient method of analysis of a spherical reflector, fed by a scalar beam produced by a complex source-point feed, is presented. Two cases, soft and hard reflector surface, are considered. In each case the solution of the full-wave integral equation is reduced to dual series equations and then further to a regularized infinite-matrix equation. The latter procedure is based on the analytical inversion of the static part of the problem. Sample numerical results for $50-\lambda$ reflectors demonstrate features that escape a high-frequency asymptotic analysis. (C) 2000 Acoustical Society of America. [S0001-4966(00)00306-4]
\end{abstract}

PACS numbers: 43.20.Fn [ANN]

\section{INTRODUCTION}

Most frequently, performance of a reflector antenna is predicted by using asymptotic high-frequency techniques such as Physical Optics (PO), combined with Geometrical Theory of Diffraction (GTD), ${ }^{1,2}$ for the off-beam radiation. The Method-of-Moments (MoM) is also used in the integralequation (IE) analysis of reflectors of small to moderate size in terms of wavelength. ${ }^{3,4}$ The merits and limitations of both approaches are well known. In spite of their flexibility, PO or GTD alone is not uniformly accurate with respect to the direction in space, and both fail to characterize smaller reflectors. MoM algorithms for the full-wave IE become computationally expensive for larger reflectors, due to either large matrices or a large time for filling the matrix. Besides, not every MoM approximation scheme is convergent to the exact result as the number of equations is increased, in the sense that the computation error cannot be progressively minimized.

The feed field is normally simulated via a Gaussian beam or a spherical-wave expansion multiplied with an angular window function. Commonly it is neglected by these simulations that such a feed field function does not solve the Helmholtz equation exactly, although the radiated or scattered field is found as a solution of the full-wave integral equation. It has been proposed therefore to use the complex source-point (CSP) beam, or combination of such beams, as a feed field. ${ }^{5,6}$ Then, the latter is an exact solution to the Helmholtz equation at every point in the physical observation space. In Refs. 7, 8, this concept was combined with PO and GTD for a characterization of a spherical-wave scalar

\footnotetext{
${ }^{a}$ Visiting Scientist from Kharkov Academy of Civil Engineering, Kharkov 310002, Ukraine.

${ }^{b}$ Visiting Scientist from Institute of Radiophysics and Electronics NAS, Kharkov 310085, Ukraine.
}

beam scattering from a circular aperture. This concept is further developed in Refs. 9, 10, which contain practical and useful results.

In a recent study, ${ }^{11}$ it was demonstrated, for a $2 \mathrm{D}$ problem, that a very accurate and computationally efficient analysis of reflector antennas can be achieved by using a combination of the CSP method to simulate a beam-like feed field, and an analytical regularization based technique to solve the reflector scattering. In Ref. 11, a circular-cylindrical reflector fed by the CSP-type line sources was considered. First, the IE was discretized into the dual series equations (DSE) in terms of the entire-period angular exponents. Then, the static part of DSE was analytically inverted, using the RiemannHilbert Problem solution, resulting in an infinite matrix Fredholm equation of the second kind. A remarkable feature of this equation is that it can be solved numerically with a guaranteed accuracy and small CPU time expenditures.

Our present study is similar to Ref. 11, but deals with a quasi-3D problem of a spherical reflector. Acoustic reflectors of this type have been analyzed in Refs. 12, 13 assuming finite transparency of reflector material and tapered spherical wave illumination. Unlike Refs. 12, 13, we characterize the feed by a scalar CSP beam, assume the reflector to be perfectly hard or soft, and develop a numerically exact solution. It is obtained from the DSE in terms of Legendre polynomials, with the static part inversion based on the Abel integral equation technique. ${ }^{14,15}$ This solution is equivalent to a judicious choice of the expansion functions in an MoM procedure, forming a set of the orthogonal eigenfunctions of the static limit of the IE kernel. The usage of a directive CSP field as a feed brings new features, hence we believe that an in-depth physical analysis of two scalar problems of soft and hard reflectors is reasonable. To obtain the results which are applicable to paraboloidal reflectors as well, we restrict the computed examples to "dish" reflectors with a large to mod- 


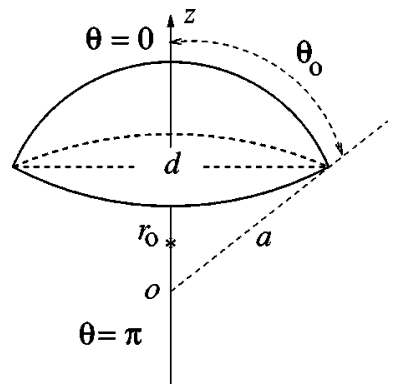

FIG. 1. Geometry of a spherical reflector fed by a CSP feed.

erate $f / d$ ratio [ $f$ and $d$ being the Geometrical Optics (GO) focal distance and the dish diameter, respectively].

The remainder of the paper is organized as follows. In Sec. I, we formulate the boundary-value problem for soft and hard reflectors, and derive a rigorous IE for each case. Further we discretize and convert it to the DSE. In Sec. II, we present basic points of the partial inversion of the DSE and reduction to the regularized matrix equation. Section III contains the formulas for far field characteristics. Section IV presents the results of numerical analysis concerning radiation patterns and the directivity of the soft and hard spherical reflectors. Conclusions of the presented work are summarized in Sec. V. Focal shifts in parabolic reflectors. A note should be made that the time dependence is assumed as $e^{-i \omega t}$ and is omitted.

\section{FORMULATION AND BASIC EQUATIONS}

\section{A. Problem formulation}

Consider a zero-thickness, perfectly soft or perfectly hard, spherical reflector of radius $a$ and angular width $2 \theta_{0}$, symmetrically excited by the field of a CSP beam. That is, the feed is located at the point $\left(r_{s}, 0,0\right)$ with the radial source coordinate being a complex value: $r_{s}=r_{0}+i b$. The geometry of the problem is shown in Fig. 1. The incident scalar wave field is:

$$
U^{0}(r, \theta)=e^{i k R} / R,
$$

where $R=\left(r^{2}-2 r r_{s} \cos \theta+r_{s}^{2}\right)^{1 / 2}$, and $k=\omega / c$ is the realvalued free-space wave number ( $c$ being the sound propagation velocity). The scattered field $U^{\mathrm{sc}}(r, \theta)$ is the solution of a boundary-value problem for the 3D Helmholtz equation, with the boundary condition of either soft or hard type at the reflector surface $M:\left(r=a, 0 \leqslant \theta<\theta_{0}, 0 \leqslant \varphi \leqslant 2 \pi\right)$

$$
U^{0}+\left.U^{\mathrm{sc}}\right|_{M}=0,\left.\quad \frac{\partial\left(U^{0}+U^{\mathrm{sc}}\right)}{\partial r}\right|_{M}=0 .
$$

The formulation must also include (i) the edge condition $U^{\mathrm{sc}} \sim O\left(\rho^{1 / 2}\right), \partial U^{\mathrm{sc}} / \partial r \sim O\left(\rho^{-1 / 2}\right)$, where $\rho \rightarrow 0$ is the distance from the dish rim, and (ii) the outgoing radiation condition at $r \rightarrow \infty$, to ensure the solution uniqueness. ${ }^{16}$

\section{B. Acoustically soft reflector}

Consider first the case of a perfectly soft reflector. We seek the scattered field function as a single-layer potential:

$$
U^{\mathrm{sc}}(\mathbf{r})=-\frac{1}{4 \pi} \iint_{M} j_{S}\left(\theta^{\prime}, \varphi^{\prime}\right) \frac{e^{i k\left|\mathbf{r}-\mathbf{r}^{\prime}\right|}}{\left|\mathbf{r}-\mathbf{r}^{\prime}\right|} d S^{\prime},
$$

where $\quad\left|\mathbf{r}-\mathbf{r}^{\prime}\right|=\left\{r^{2}+a^{2}-2 r a\left[\cos \theta^{\prime} \cos \theta+\sin \theta^{\prime} \sin \theta\right.\right.$ $\left.\left.\times \cos \left(\varphi-\varphi^{\prime}\right)\right]\right\}^{1 / 2}$ is the distance between the observation point and a point at the reflector surface. Note that the unknown density function is related to the jump of the scattered field normal derivative across the reflector as

$$
j_{s}\left(\theta^{\prime}, \varphi^{\prime}\right)=\left.\frac{\partial U^{\mathrm{sc}}}{\partial r}\right|_{r=a+0}-\left.\frac{\partial U^{\mathrm{sc}}}{\partial r}\right|_{r=a-0} .
$$

The soft-surface boundary condition Eq. (2) then yields a Fredholm first kind IE as

$$
\begin{aligned}
& \frac{a}{8 \pi} \int_{0}^{\theta_{0}} \int_{0}^{2 \pi} j_{s}\left(\theta^{\prime}, \varphi^{\prime}\right) \frac{e^{i 2 k a|\sin (\psi / 2)|}}{|\sin (\psi / 2)|} \sin \theta^{\prime} d \theta^{\prime} d \varphi^{\prime} \\
& \quad=U^{0}(a, \theta, \varphi),
\end{aligned}
$$

where $\psi=\arccos \left[\cos \theta^{\prime} \cos \theta+\sin \theta^{\prime} \sin \theta \cos \left(\varphi-\varphi^{\prime}\right)\right]$.

Instead of approximating IE Eq. (5) by MoM with subdomain or $M$-domain basis functions, we further discretize it in terms of a complete set of orthogonal functions in the global domain $0 \leqslant \theta \leqslant \pi$. In our case of a $\varphi$-independent solution, such a set is formed by the Legendre polynomials $P_{n}(\cos \theta)(n=0,1,2, \ldots)$. By extending the density function to be identically by zero on the complementary surface ( $r$ $\left.=a, \theta_{0}<\theta \leqslant \pi\right)$, as is natural due to Eq. (4), we assume that for all $\theta^{\prime}$

$$
j_{s}\left(\theta^{\prime}\right)=-\frac{1}{k a^{2}} \sum_{n=0}^{\infty} x_{n}^{s}(2 n+1) P_{n}\left(\cos \theta^{\prime}\right),
$$

with the expansion coefficients $x_{n}^{s}, n=0,1,2, \ldots$ to be found.

Besides, it is known that the free-space Green's function can be expanded as: ${ }^{15}$

$$
\begin{aligned}
\frac{e^{i k\left|\mathbf{r}-\mathbf{r}^{\prime}\right|}}{\left|\mathbf{r}-\mathbf{r}^{\prime}\right|}= & i k \sum_{m=0}^{\infty}\left(2-\delta_{0 m}\right) \cos m\left(\varphi-\varphi^{\prime}\right) \\
& \times \sum_{n=m}^{\infty}(2 n+1) \frac{(n-m) !}{(n+m) !} \\
& \times\left\{\begin{array}{ll}
h_{n}^{(1)}(k a) j_{n}(k r), & r<a \\
j_{n}(k a) h_{n}^{(1)}(k r), & r>a
\end{array}\right\} \\
& \times P_{n}^{m}(\cos \theta) P_{n}^{m}\left(\cos \theta^{\prime}\right),
\end{aligned}
$$

where $P_{n}^{m}$ are the associated Legendre functions. This yields the following series representation of the kernel function of the IE Eq. (5):

$$
\begin{aligned}
\frac{e^{i 2 k a|\sin (\psi / 2)|}}{|\sin (\psi / 2)|}= & i 2 k a \sum_{m=0}^{\infty}\left(2-\delta_{0 m}\right) \cos m\left(\varphi-\varphi^{\prime}\right) \\
& \times \sum_{n=m}^{\infty}(2 n+1) \frac{(n-m) !}{(n+m) !} j_{n}(k a) h_{n}^{(1)}(k a) \\
& \times P_{n}^{m}(\cos \theta) P_{n}^{m}\left(\cos \theta^{\prime}\right) .
\end{aligned}
$$


Now the integration in Eq. (5) over $\theta^{\prime}$ can be extended from 0 to $\pi$. This enables us to use orthogonality of the spherical harmonics

$$
\begin{gathered}
\int_{0}^{2 \pi} \cos m\left(\varphi-\varphi^{\prime}\right) \int_{0}^{\pi} P_{s}\left(\cos \theta^{\prime}\right) P_{n}\left(\cos \theta^{\prime}\right) \sin \theta^{\prime} d \theta^{\prime} d \varphi^{\prime} \\
\quad=\frac{4 \pi(s+m) !}{(2 n+1))(s-m) !} \delta_{n s} \delta_{m 0}
\end{gathered}
$$

when discretizing the IE. Together with the specification that the density function Eq. (6) is zero off the reflector, this brings us to the dual series equations (DSE):

$$
\begin{gathered}
\sum_{n=0}^{\infty} x_{n}^{s}(2 n+1) j_{n}(k a) h_{n}^{(1)}(k a) P_{n}(\cos \theta) \\
=\sum_{n=0}^{\infty} b_{n}^{s} P_{n}(\cos \theta), \quad 0 \leqslant \theta<\theta_{0}, \\
\sum_{n=0}^{\infty} x_{n}^{s}(2 n+1) P_{n}(\cos \theta)=0, \quad \theta_{0}<\theta \leqslant \pi,
\end{gathered}
$$

where the right-hand side coefficients are determined by the CSP feed field as

$$
b_{n}^{s}=(2 n+1) j_{n}\left(k r_{s}\right) h_{n}^{(1)}(k a) .
$$

Once again, the DSE Eq. (10) can be attacked by a "'brute force" numerical solution with a direct MoM scheme. Although the results are generally meaningful, only a few correct digits can be obtained, and there is no possibility of increasing accuracy by taking a greater number of collocation points. That is our motivation for regularizing the DSE Eq. (10), to obtain an algorithm convergent to the exact solution in a pointwise manner.

\section{Acoustically hard reflector}

For a perfectly hard spherical reflector fed by a CSP feed, we seek the scattered field function as a double-layer potential:

$$
U^{\mathrm{sc}}(\mathbf{r})=-\frac{1}{4 \pi} \iint_{M} j_{h}\left(\theta^{\prime}, \varphi^{\prime}\right) \frac{\partial}{\partial r^{\prime}}\left\{\frac{e^{i k\left|\mathbf{r}-\mathbf{r}^{\prime}\right|}}{\left|\mathbf{r}-\mathbf{r}^{\prime}\right|}\right\} d S^{\prime},
$$

where the $\varphi$-independent density function is now related to the jump of the field across the reflector surface:

$$
j_{h}\left(\theta^{\prime}\right)=U^{\mathrm{sc}}\left(a+0, \theta^{\prime}\right)-U^{\mathrm{sc}}\left(a-0, \theta^{\prime}\right) .
$$

The hard-surface boundary condition Eq. (2) now yields a hypersingular IE for this function:

$$
\begin{aligned}
\frac{a^{2}}{4 \pi} & \frac{\partial}{\partial r} \int_{0}^{\theta_{0}} \int_{0}^{2 \pi} j_{h}\left(\theta^{\prime}, \varphi^{\prime}\right) \frac{\partial}{\partial r^{\prime}}\left\{\frac{e^{i k\left|\mathbf{r}-\mathbf{r}^{\prime}\right|}}{\left|\mathbf{r}-\mathbf{r}^{\prime}\right|}\right\} \sin \theta^{\prime} d \theta^{\prime} d \varphi^{\prime} \\
& =\frac{\partial}{\partial r} U^{0}(r, \theta) .
\end{aligned}
$$

In order to discretize the IE Eq. (14), we assume that the density function is extended, so that it is identically zero on the rest of the sphere of radius $a$; we expand the density function in terms of the same complete set of Legendre polynomials as were used in the "soft case":

$$
j_{h}\left(\theta^{\prime}\right)=\frac{1}{k^{2} a} \sum_{n=0}^{\infty} x_{n}^{h} P_{n}\left(\cos \theta^{\prime}\right) .
$$

We substitute this series into the IE Eq. (14) and integrate, making use of the orthogonality properties Eq. (9). Moreover, we explicitly enforce the vanishing of $j_{h}\left(\theta^{\prime}\right)$ off the reflector surface, and so arrive at the DSE as

$$
\begin{aligned}
& \sum_{n=0}^{\infty} x_{n}^{h} j_{n}^{\prime}(k a) h_{n}^{(1)^{\prime}}(k a) P_{n}(\cos \theta)=-\sum_{n=0}^{\infty} b_{n}^{h} P_{n}(\cos \theta), \\
& 0 \leqslant \theta<\theta_{0}, \\
& \sum_{n=0}^{\infty} x_{n}^{h} P_{n}(\cos \theta)=0, \quad \theta_{0}<\theta \leqslant \pi,
\end{aligned}
$$

where

$$
b_{n}^{h}=j_{n}\left(k r_{s}\right) h_{n}^{(1)^{\prime}}(k a) \text {. }
$$

Note that the DSE so obtained are of the same type as in the case of the soft reflector.

\section{PARTIAL INVERSION OF DSE}

We shall regularize Eqs. (10) and (16) by performing an analytical inversion of the static part of the DSE. To extract the static parts, use the power series for the spherical Bessel functions, ${ }^{17}$ from which it follows that:

$$
\begin{aligned}
& j_{n}(k a)=\frac{n !(2 k a)^{n}}{(2 n+1) !}\left\{1+O\left(\frac{k^{2} a^{2}}{n}\right)\right\}, \\
& h_{n}^{(1)}(k a)=-i \frac{(2 n) !}{n !(2 k a)^{n+1}}\left\{1+O\left(\frac{k^{2} a^{2}}{n}\right)\right\} .
\end{aligned}
$$

This enables us to show that if $n \gg k a$, then

$$
\begin{aligned}
& (2 n+1) j_{n}(k a) h_{n}^{(1)}(k a) \sim-i /(k a), \\
& (2 n+1)^{-1} j_{n}^{\prime}(k a) h_{n}^{(1)^{\prime}}(k a) \sim i /\left(4 k^{3} a^{3}\right) .
\end{aligned}
$$

Based on these estimates, we introduce two coefficient sets as

$$
\begin{aligned}
& \varepsilon_{n}^{s}=1-i k a(2 n+1) j_{n}(k a) h_{n}^{(1)}(k a), \\
& \varepsilon_{n}^{h}=1+i 4(k a)^{3}(2 n+1)^{-1} j_{n}^{\prime}(k a) h_{n}^{(1) \prime}(k a) .
\end{aligned}
$$

Note that all $\varepsilon_{n}^{s}$ behave as $O\left(k^{2} a^{2} n^{-2}\right)$ for larger $n$, or, equivalently, for smaller $k a$, while $\varepsilon_{n}^{h}$ behave as $(2 n$ $+1)^{-2}$. The DSE may be written as

$$
\begin{gathered}
\sum_{n=0}^{\infty} x_{n}^{s} P_{n}(\cos \theta)=\sum_{n=0}^{\infty}\left(\varepsilon_{n}^{s} x_{n}^{s}-i k a b_{n}^{s}\right) P_{n}(\cos \theta), \\
0 \leqslant \theta<\theta_{0}, \\
\sum_{n=0}^{\infty}(2 n+1) x_{n}^{s} P_{n}(\cos \theta)=0, \quad \theta_{0}<\theta \leqslant \pi,
\end{gathered}
$$

and 


$$
\begin{aligned}
& \sum_{n=0}^{\infty}(2 n+1) x_{n}^{h} P_{n}(\cos \theta) \\
& \quad=\sum_{n=0}^{\infty}(2 n+1)\left(x_{n}^{h} \varepsilon_{n}^{h}+i 4 k^{3} a^{3} b_{n}^{h}\right) P_{n}(\cos \theta), \\
& \quad 0 \leqslant \theta<\theta_{0}, \\
& \sum_{n=0}^{\infty} x_{n}^{h} P_{n}(\cos \theta)=0, \quad \theta_{0}<\theta \leqslant \pi .
\end{aligned}
$$

Analytical inversion of the left-hand side the DSE is performed by transforming it to a single function, defined on the complete interval $[0, \pi]$ of $\theta$ variation. This is done by reducing each of the functional equations of the DSE to an Abel IE (see Refs. 12, 13), which has a known inversion formula. Here we use the Mehler-Dirichlet formulas for the Legendre polynomials:

$$
\begin{aligned}
P_{n}(\cos \theta) & =\int_{0}^{\theta} \frac{\cos (n+1 / 2) \gamma}{(\cos \gamma-\cos \theta)^{1 / 2}} d \gamma \\
& =\int_{\theta}^{\pi} \frac{\sin (n+1 / 2) \gamma}{(\cos \theta-\cos \gamma)^{1 / 2}} d \gamma .
\end{aligned}
$$

This enables us to integrate the second equation of Eqs. (21) and the first of Eqs. (22), and reduce each of the DSE to the same function of $\theta$, given by its piecewise Fourierexpansion on $[0, \pi]$ :

$$
\begin{aligned}
& \sum_{n=0}^{\infty} x_{n}^{s} \cos (n+1 / 2) \theta \\
& \quad=\left\{\begin{array}{l}
\sum_{n=0}^{\infty}\left(x_{n}^{s} \varepsilon_{n}^{s}+B_{n}^{s}\right) \cos (n+1 / 2) \theta, \quad 0 \leqslant \theta<\theta_{0} \\
0, \quad \theta_{0}<\theta \leqslant \pi
\end{array}\right. \\
& \sum_{n=0}^{\infty} x_{n}^{h} \sin (n+1 / 2) \theta \\
& =\left\{\begin{array}{l}
\infty \\
\sum_{n=0}^{\infty}\left(x_{n}^{h} \varepsilon_{n}^{h}+B_{n}^{h}\right) \sin (n+1 / 2) \theta, \quad 0 \leqslant \theta<\theta_{0} \\
0, \quad \theta_{0}<\theta \leqslant \pi
\end{array}\right.
\end{aligned}
$$

Here we have denoted

$$
B_{n}^{s}=-i k a b_{n}^{s}, \quad B_{n}^{h}=i 4(k a)^{3} b_{n}^{h} .
$$

Using orthogonality and completeness of the functions $\cos (n+1 / 2) \theta$ or $\sin (n+1 / 2) \theta, n=(0), 1,2, \ldots$ at the interval $(0, \pi)$, produces a regularized infinite-matrix equation:

$$
x_{m}^{h, s}=\frac{1}{\pi} \sum_{n=0}^{\infty}\left(x_{n}^{h, s} \varepsilon_{n}^{h, s}+B_{n}^{h, s}\right) S_{m n}^{s, h}\left(\theta_{0}\right), \quad m=0,1,2, \ldots,
$$

where

$$
S_{m n}^{s, h}\left(\theta_{0}\right)=\frac{\sin (n-m) \theta_{0}}{n-m} \pm \frac{\sin (n+m+1) \theta_{0}}{n+m+1}
$$

with the upper and lower sign for the soft and hard case, respectively.

Taking account of the large-index behavior of $\varepsilon_{n}^{s}$ or $\varepsilon_{n}^{h}$, it is easy to verify that the absolute squared norm $N_{A}^{2}$ $=\sum_{m, n=0}^{\infty}\left|\varepsilon_{n}^{s, h}(k a) S_{m n}^{s, h}\left(\theta_{0}\right)\right|^{2} \leqslant$ const $\sum_{n=0}^{\infty}\left|\varepsilon_{n}^{s, h}\right|^{2}$ is finite. This is enough to conclude that the matrix operator of Eq. (27) is of Fredholm second kind type in the space of the square summable sequences $l^{2}$. Besides, the right-hand side of Eq. (27) belongs to $l^{2}$, provided that $\left|r_{s}\right|<a$, or, more precisely, if the real-space branch-cut associated with the CSP feed (see Refs. 5-8) does not touch or cross the reflector. Under such a condition, the Fredholm theorems are valid: ${ }^{16}$ due to uniqueness, the exact solution of the infinitematrix equation (27) exists in $l^{2}$. Moreover, it can be shown that the solution satisfies the edge condition: without going into details we point out the connection with the square-root denominators in the integrands of Eq. (23). For computational purposes the most important consequence of the regularization procedure is that, the greater the truncation order of Eq. (27), the closer the numerical solution will be to the exact one. The convergence here is of pointwise-type, not of mean-type, or of some other "weak" form. Note that the matrix elements are remarkably simple, and need no numerical integrations. If $k=0$, all the coefficients $\varepsilon_{n}^{s}$ vanish, showing that in the static case Eq. (27) delivers an exact analytical solution. In the case of the hard boundary condition, $\varepsilon_{n}^{h}$ $=(2 n+1)^{-2}$ in the limit $k \rightarrow 0$. This means that by introducing new coefficients $\widetilde{\varepsilon}_{n}^{h}=\varepsilon_{n}^{h}-(2 n+1)^{-2}$, one can also obtain an exact analytical solution of the static-counterpart of the hard-surface problem. However, as spherical reflectors are normally used with $k a \gg 1$, such a procedure is not necessary in our analysis.

\section{FAR FIELD CHARACTERISTICS}

After determining the coefficients $x_{n}^{s, h}$ from Eq. (27), one can easily find, with the same guaranteed accuracy, the density function, the far field pattern, the total radiated power, and the directivity (which, in our lossless analysis, is the same as gain). All of these functions and parameters are expressed in terms of series depending on $x_{n}^{s, h}$. For example, the far field pattern is found as

$$
\Psi^{s, h}(\theta)=e^{k\left(b-i r_{0}\right) \cos \theta}+\sum_{n=0}^{\infty}(-i)^{n} w_{n}^{s, h} y_{n}^{s, h} P_{n}(\cos \theta),
$$

where we denote $y_{n}^{s}=j_{n}(k a) x_{n}^{s}, y_{n}^{h}=j_{n}^{\prime}(k a) x_{n}^{h}$, and $w_{n}^{s}$ $=2 n+1, w_{n}^{h}=1$.

Due to completeness and orthogonality of the expansion functions on the unit sphere, integration of the time-average far-zone power flux is performed analytically, yielding

$$
\begin{aligned}
P_{\text {tot }}^{s}= & P_{0}+P_{0} \frac{2 k b}{\sinh 2 k b} \\
& \times \sum_{n=0}^{\infty}(2 n+1)\left\{\left|y_{n}^{s}\right|^{2}+2 \operatorname{Re}\left[y_{n}^{s} j_{n}\left(k r_{s}\right)\right]\right\},
\end{aligned}
$$




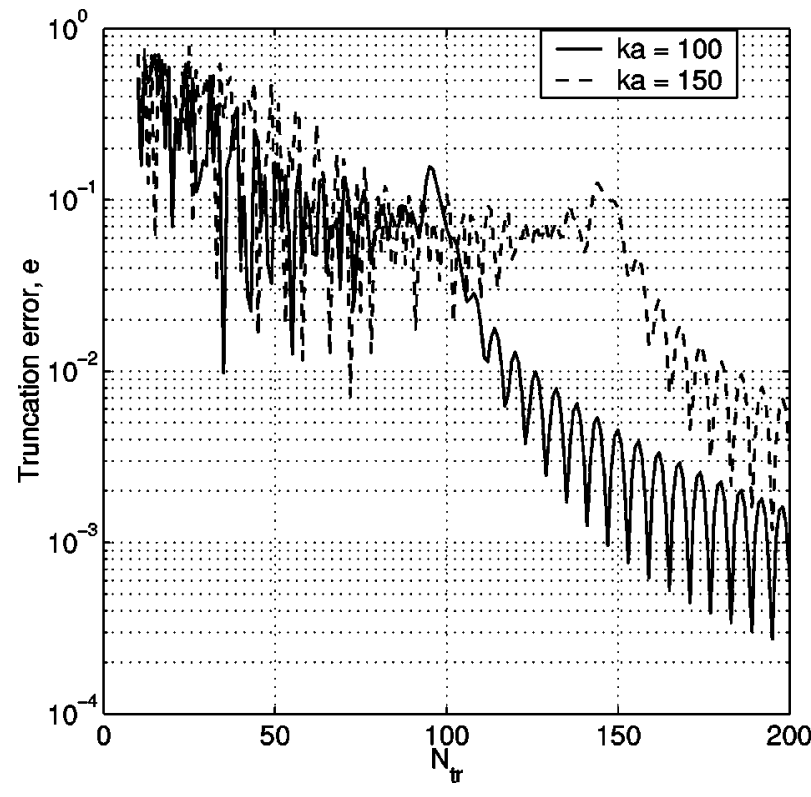

FIG. 2. Normalized computation error as a function of the matrix truncation number, for a hard-surface reflector $\theta_{0}=30^{\circ}$.

$$
\begin{aligned}
P_{\text {tot }}^{h}= & P_{0}+P_{0} \frac{2 k b}{\sinh 2 k b} \sum_{n=0}^{\infty}\left\{(2 n+1)^{-1}\left|y_{n}^{h}\right|^{2}\right. \\
& \left.+2 \operatorname{Re}\left[y_{n}^{h} j_{n}\left(k r_{s}\right)\right]\right\} .
\end{aligned}
$$

The directivity is

$$
D_{\mathrm{tot}}^{s, h}=\frac{4 \pi}{k^{2} P_{\mathrm{tot}}^{s, h}}\left|e^{i k r_{s}}+\sum_{n=0}^{\infty} i^{n} y_{n}^{s, h} w_{n}^{s, h}\right|^{2} .
$$

Note that the free-space radiated power and the freespace directivity of the same CSP feed are given, respectively, by the expressions:

$$
P_{0}=\frac{2 \pi}{k^{2}} \frac{\sinh 2 k b}{k b}, \quad D_{0}=\frac{2 k b e^{2 k b}}{\sinh 2 k b} .
$$

Overall directivity $D_{\text {tot }}$ should be compared with $D_{0}$.

\section{NUMERICAL RESULTS}

In principle, the accuracy in solving Eq. (27) is limited only by the digital precision of the computer used, in contrast to the conventional MoM-type numerical approximations (e.g., Refs. 3, 4). For an accuracy in the far field of three digits, and in the near field of two digits, the number of equations to be taken is $N_{\mathrm{tr}} \geqslant k a+20$ independently of the angular width $2 \theta_{0}$, and of the feed parameters. This estimate is illustrated by the plots of normalized error of computed density function versus truncation number, presented in Fig. 2 (for the hard-surface case). The error is computed in the maximum-norm sense:

$$
e(N)=\frac{\max _{n \leqslant N}\left|x_{n}^{N+1}-x_{n}^{N}\right|}{\max _{n \leqslant N}\left|x_{n}^{N}\right|} .
$$

However, the error computed in the $l^{2}$ sense shows very similar behavior. It should be recalled that the Fredholm nature of Eq. (26) guarantees that $e(N) \rightarrow 0$ as $N \rightarrow \infty$. CPU

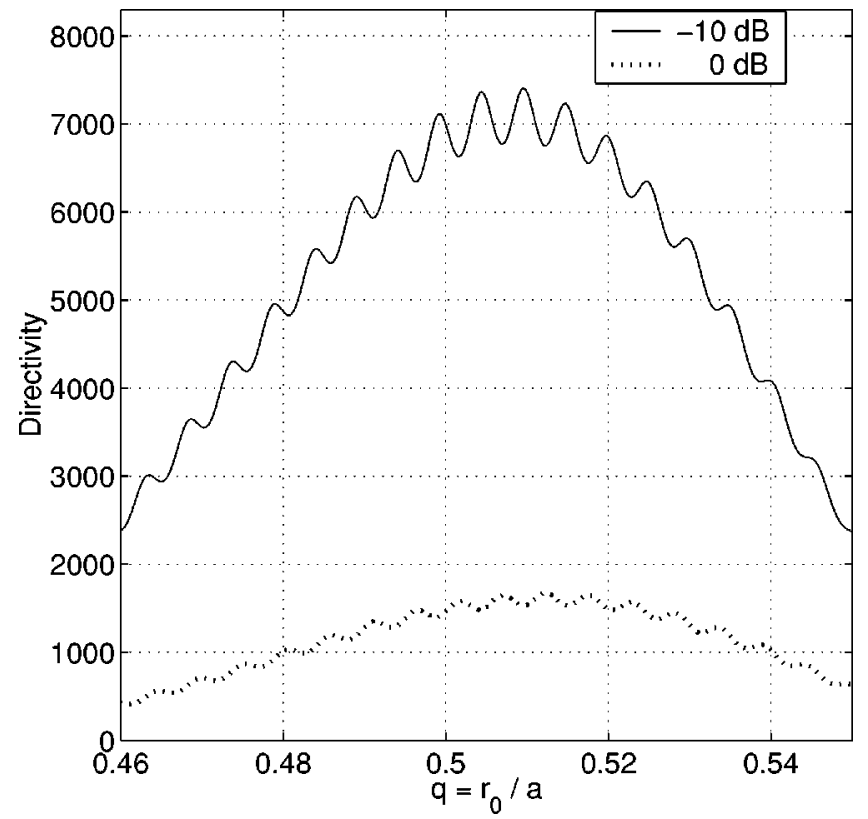

FIG. 3. Directivity of a hard-surface "shallow" reflector as a function of the normalized feed position. Solid curve is for the edge illumination -10 $\mathrm{dB}$, dashed curve is for nondirective source $(k b=0)$. Reflector parameters are: $d=50 \lambda, f / d=0.97\left(k a=606.9, \theta_{0}=15^{\circ}\right)$.

time needed for solving a 50- $\lambda$ reflector of angular halfwidth $\theta_{0}=15^{\circ}$, that is $k a=620$, with a Pentium 133 computer and Fortran 77 source-code under Windows 3.11 was 2.5 min.

We present some further results on the dependence of overall directivity on the feed location in real space. We examine two $d=50 \lambda$ hard-surface reflectors: a "shallow dish" $\left(\theta_{0}=15^{\circ}\right.$, or $\left.f / d=0.97\right)$ and a 'deep', one $\left(\theta_{0}\right.$ $=30^{\circ}$, or $\left.f / d=0.5\right)$. The spherical reflector is believed to behave as a paraboloidal one, provided that the geometrical deviation between the two surfaces does not exceed $\lambda / 16$ (or even $\lambda / 8) .{ }^{1}$ Despite the paper titles, this is why spherical reflectors were considered in Refs. 12, 13. According to Fig. 2 of Ref. 11, this limits the aperture size of our "shallow dish" to the value $d=53.5 \lambda$. Of course, even deeper or larger reflectors can still be considered (there is no computational difficulty), but spherical aberrations are known to degrade the main beam. This happens in the case of our "deep" reflector. However, such spherical reflectors remain of interest, due to easier manufacturing and mechanical beam steering.

Only an infinite paraboloid generates a plane wave, if the point feed is placed at the GO focus: $r_{0} / a=0.5$. In any finite-size geometry a focal shift occurs, predicted by $\mathrm{GO},{ }^{18}$ and studied in Ref. 19 by using PO. However in reality, for a finite $d$, the dependence of $D_{\text {tot }}$ on $q=r_{0} / a$ has an even more complex and oscillatory nature. In Fig. 3 is presented the hard "shallow" reflector under two edge illuminations: $-10 \mathrm{~dB}$ (the same for all $q$, solid curve) and $0 \mathrm{~dB}$ (omnidirectional source, dashed curve). In the former case, the parameter $k b$ was slightly varied around the value 8.2 to provide a constant illumination level. One may clearly see that there is not a single, but several positions of the feed providing almost equally good directivity. The broad maximum corresponds to the focal shift predicted by $\mathrm{GO}:{ }^{19}$ it is between $r_{0} / a=0.5$ and $r_{0} / a=\sec \theta_{0} / 2$, that is $r_{0} / a=0.518$ 


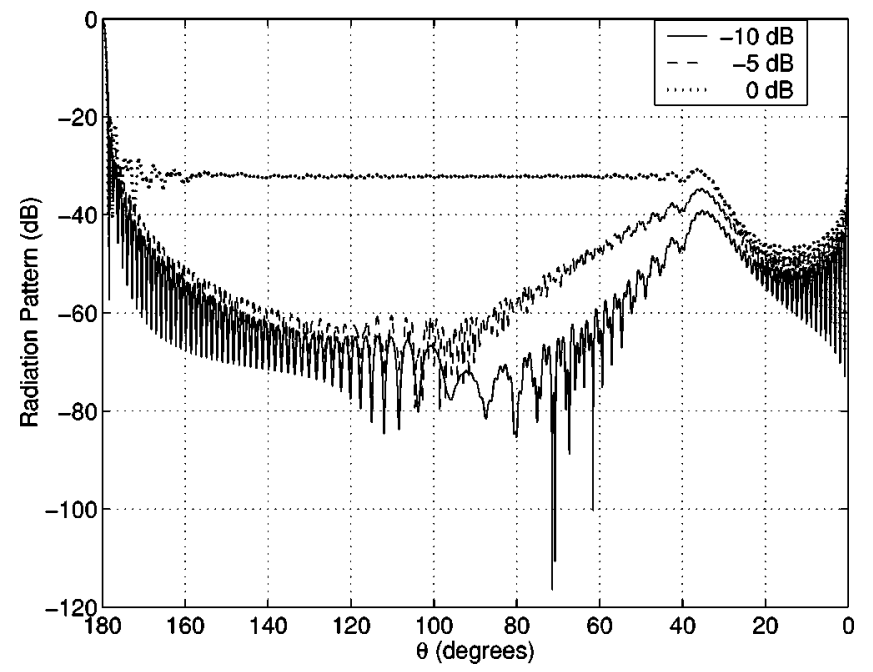

FIG. 4. Normalized far field radiation patterns of the same "shallow" reflector as in Fig. 3, for the feed position $r_{0} / a=0.507$. Edge illuminations are: $-10 \mathrm{~dB}$ (solid curve), $-5 \mathrm{~dB}$ (dashed curve), and non-directive source (dotted curve).

here. The period of the smaller oscillations is equal to $\lambda / 2$. This feature of the near field is clearly not of GO nature, and does not appear to be predicted by high-frequency asymptotic approximations. In Fig. 4, we present total far field radiation patterns, computed for three different edge illuminations. Note that reduction of the edge illumination levels from $-5 \mathrm{~dB}$ to $-10 \mathrm{~dB}$ (that is, increasing the beamwidth parameter $k b$, from 4 to 8.4) mainly affects the far sidelobes, between $30^{\circ}$ and $90^{\circ}$. The feed position here corresponds to the optimum.

The next series of results illustrates features of the "deep" reflector. In this case, the spherical shape of the latter has a greater effect. The directivity dependence on the feed position (Fig. 5) shows a broad maximum in the middle

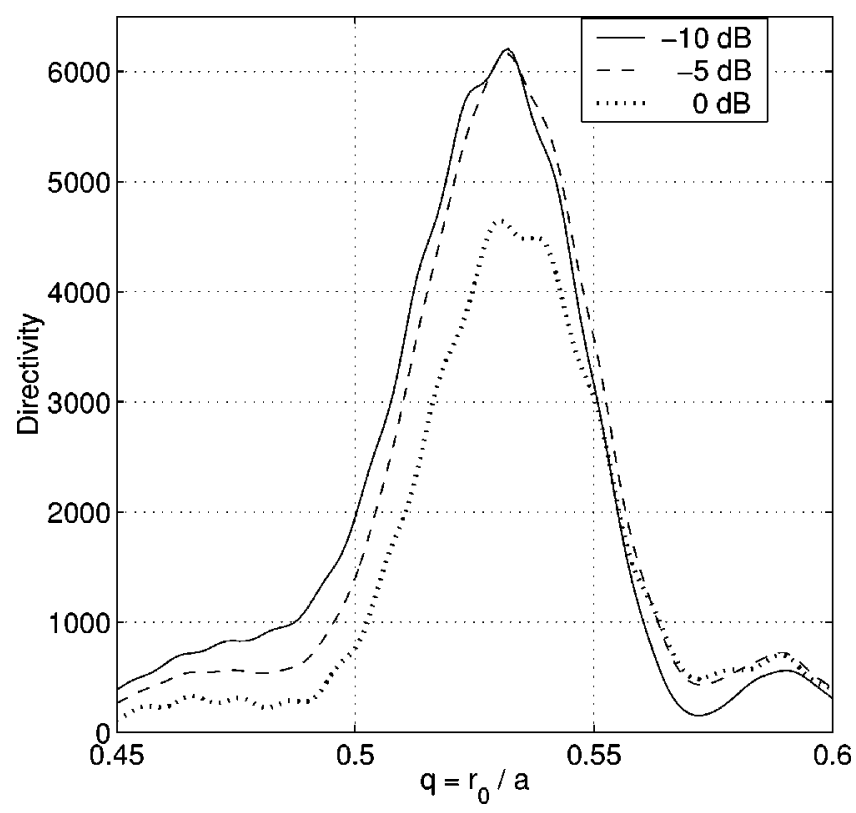

FIG. 5. Directivity of a hard-surface "deep" reflector as a function of the normalized feed position. Edge illuminations are: $-10 \mathrm{~dB}$ (solid curve), $-5 \mathrm{~dB}$ (dashed curve), and nondirective source (dotted curve). Reflector parameters are: $d=50 \lambda, f / d=0.5\left(k a=314.2, \theta_{0}=30^{\circ}\right)$.

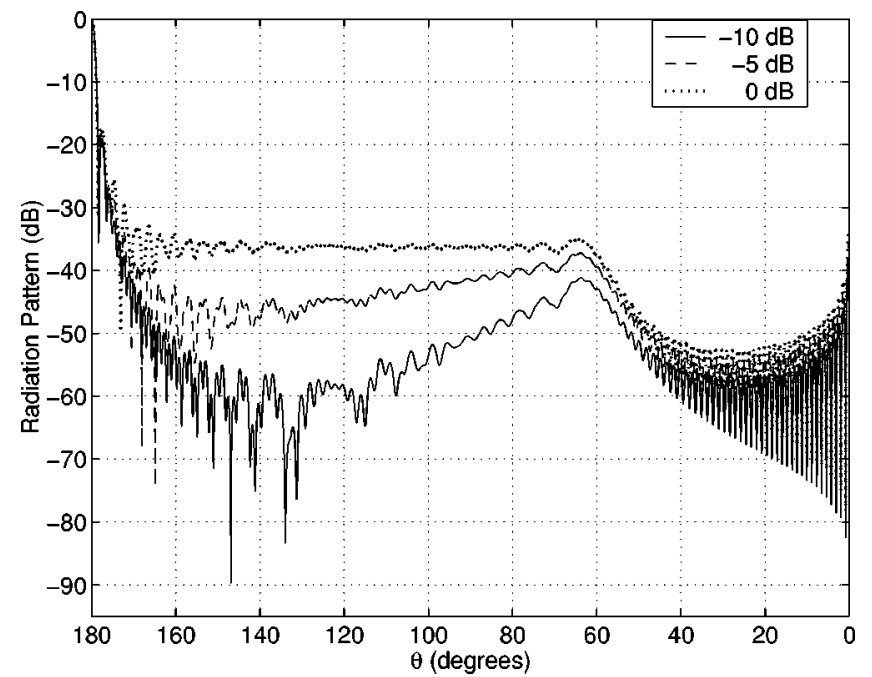

FIG. 6. Normalized far field radiation patterns of the same "deep" reflector as in Fig. 5, for the feed position $r_{0} / a=0.526$. Edge illuminations are: $-10 \mathrm{~dB}$ (solid curve), $-5 \mathrm{~dB}$ (dashed curve), and nondirective source (dotted curve).

of GO-predicted interval, between $r_{0} / a=0.5$ and 0.578 , but no oscillations. Note that the maximum directivity for the omnidirectional feed is double that of the "shallow" reflector case (compare with Fig. 3), due to doubling the area of reflector. However, the directivity in this case is much more critically dependent on the feed position, the GO focus being completely unacceptable. Far field patterns presented in Fig. 6 are more sensitive to the decrease of edge illumination levels from $-5 \mathrm{~dB}$ to $-10 \mathrm{~dB}$ (by increasing the beamwidth parameter $k b$, from 0.8 to 2.1 ), and show the effect of spherical aberrations, not visible in Fig. 4.

Most of these features are observed for the soft reflectors as well: see Figs. 7 and 8. Similarly for the hard reflector,

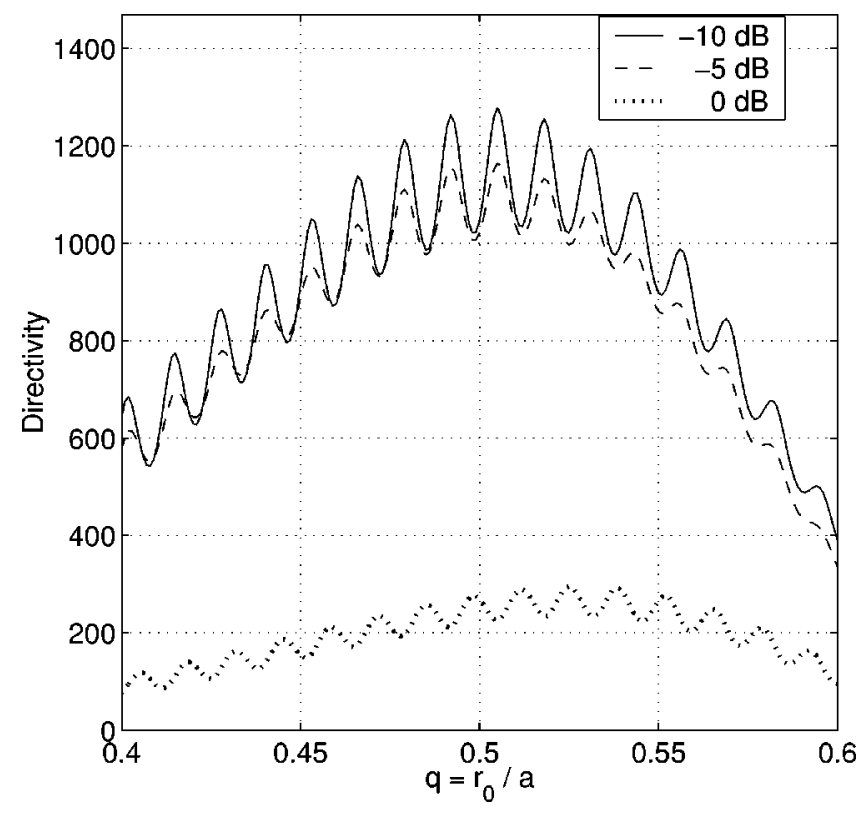

FIG. 7. Directivity of a soft-surface "shallow" reflector as a function of the normalized feed position. Edge illuminations are: $-10 \mathrm{~dB}$ (solid curve), and nondirective source (dotted curve). Reflector parameters are: $d=20 \lambda, f / d$ $=0.97\left(k a=242.8, \theta_{0}=15^{\circ}\right)$. 


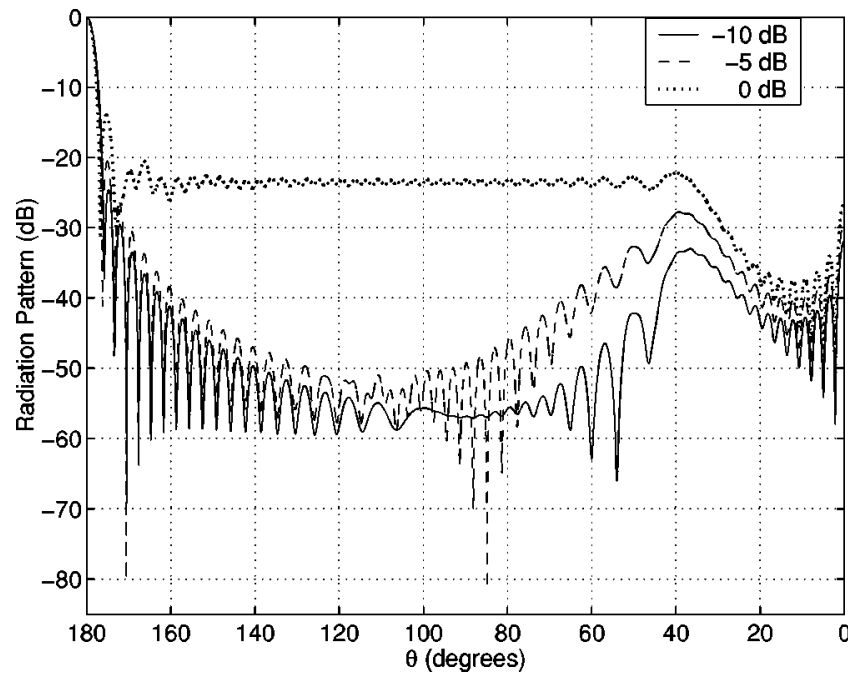

FIG. 8. Normalized far field radiation patterns of the same reflector as in Fig. 7, for the feed position $r_{0} / a=0.504$. Edge illuminations are: $-10 \mathrm{~dB}$ (solid curve), $-5 \mathrm{~dB}$ (dashed curve), and nondirective source (dotted curve).

decreasing the edge illumination below $-5 \mathrm{~dB}$ has a small effect on the overall directivity. As expected, a soft-surface edge produces smaller sidelobes than a hard-surface one.

\section{CONCLUSIONS}

We have presented a simple but powerful algorithm for the analysis of spherical reflector antennas fed by a scalar beam. It is based on the exact analytical inversion of the singular part of the corresponding full-wave integral equation. This is achieved through the conversion of the problem to dual series equations, and followed by the Abel integral transform to invert the static part (in the soft case), or the "main" part (in the hard case). The resulting infinite matrix equations are of Fredholm second kind, thus ensuring existence of the exact solution, and the opportunity of obtaining it within machine precision, by taking more and more equations. This approach was previously used in plane-wave scattering analysis; here, it is combined with a complex sourcepoint simulation of a directive feed field employing a function which is an exact solution to the Helmholtz equation. The analytical regularization approach described above can be considered numerically exact because the achievable accuracy is limited only by the computer used, and is uniform with respect to the frequency and the other parameters.

Accurate numerical analysis of the considered problem reveals several interesting features of the wave field that do not seem to be predicted by asymptotic techniques. For example, besides the well-known focal shift toward the reflector, the directivity (as a function of the feed position) displays not a single, but several, local maxima near the GOpredicted shifted focal point. Generally, optimization of antenna geometry depends on the cost functional. Maximum directivity is a possible choice of cost functional, but minimum sidelobe level is another, or a combination of both is also reasonable. Potential applications of the analysis presented are in the area of hydro-acoustic antenna design.

One possible extension of this analysis is to parabolic or other nonspherical reflectors. Here, an IE, similar to Eq. (5) or (14), will require a modified domain of integration. However, analytical regularization of this IE can be based upon the extraction and inversion of the singular static part of the spherical dish IE operator. Hence, the technique presented above will be at the core of the modified analysis.

\section{ACKNOWLEDGMENTS}

The first two authors are grateful to NATO and the Royal Society for a research fellowship which supported this work, and also to the Department of Mathematics, University of Dundee, for its support for this work.

${ }^{1}$ J. D. Kraus, Antennas (McGraw-Hill, New York, 1988).

${ }^{2}$ C. Scott, Modern Methods of Reflector Antenna Analysis and Design (Artech House, Boston, 1990).

${ }^{3}$ D. C. Jenn, M. A. Morgan, and R. J. Pogorzelski, "Characteristics of approximate numerical modeling techniques applied to resonance-sized reflectors," Electromagnetics 15, 41-53 (1995).

${ }^{4}$ M. R. Barclay and W. V. T. Rusch, " Moment-method analysis of large, axially symmetric reflector antennas using entire-domain functions," IEEE Trans. Antennas Propag. 39, 491-496 (1991).

${ }^{5}$ G. A. Deschamps, "Gaussian beam as a bundle of complex rays," Electron. Lett. 7, 684-685 (1971).

${ }^{6}$ L. B. Felsen, "Complex-point source solutions of the field equations and their relation to the propagation and scattering of Gaussian beams," Symp. Math. Instituta di Alta Matematica 18, 39-56 (1975).

${ }^{7}$ P. A. Belanger and M. Couture, "Boundary diffraction of an inhomogeneous wave," J. Opt. Soc. Am. 73, 446-450 (1983).

${ }^{8}$ G. A. Suedan and E. V. Jull, "Scalar beam diffraction by a wide circular aperture," J. Opt. Soc. Am. A 5, 1629-1634 (1988).

${ }^{9}$ A. N. Norris, "Complex point-source representation of real point sources and the Gaussian beam summation method," J. Opt. Soc. Am. A 3, 20052010 (1986).

${ }^{10}$ A. N. Norris and T. B. Hansen, "Exact complex source representations of time-harmonic radiation," Wave Motion 25, 127-141 (1997).

${ }^{11}$ T. Oğuzer, A. Altintaş, and A. I. Nosich, "Accurate simulation of reflector antennas by the complex source-dual series approach," IEEE Trans. Antennas Propag. 43, 793-801 (1995).

${ }^{12}$ Y. N. Feld and A. K. Ansryan, "Diffraction of scalar wave by a parabolic reflector,'” Radio Eng. Electron. Phys. 22, 1 (1977).

${ }^{13}$ I. V. Vovk, "Diffraction of sound waves by a parabolic reflector," Sov. Phys. Acoust. 25, 287-289 (1979).

${ }^{14}$ S. S. Vinogradov, "A soft spherical cap in the field of a plane sound wave," USSR Comput. Math. Math. Phys. 18, 244-249 (1978).

${ }^{15}$ S. S. Vinogradov, Y. A. Tuchkin, and V. P. Shestopalov, "Summatory equations with kernels in the form of Jacobi polynomials," Sov. Phys. Dokl. 25, 531-532 (1980).

${ }^{16}$ D. Colton and R. Kress, Integral Equation Methods in Scattering Theory (Wiley-Interscience, New York, 1983).

${ }^{17}$ M. Abramowitz and I. A. Stegun, Handbook of Mathematical Functions (Dover, New York, 1965).

${ }^{18}$ R. C. Spenser and G. Hyde, "Studies of the focal region of a spherical reflector: Geometrical optics," IEEE Trans. Antennas Propag. AP-16, 317-324 (1968).

${ }^{19}$ H. Ling, S.-W. Lee, P. T. C. Lam, and W. V. T. Rusch, “ Focal shifts in parabolic reflectors," IEEE Trans. Antennas Propag. AP-33, 744-748 (1985). 